\title{
La competencia digital en el docente universitario
}

\section{The Digital Competence in the University Teacher}

\author{
Yolvi Ocaña-Fernández*
}

Universidad César Vallejo, Lima, Perú

ORCID: https://orcid.org/0000-0002-2566-6875

\section{Luis Valenzuela-Fernández}

Universidad César Vallejo, Lima, Perú

ORCID: https://orcid.org/0000-0002-8743-4092

John Morillo-Flores

Universidad Continental, Lima, Perú

ORCID: https://orcid.org/0000-0002-2136-4458

Recibido 15-08-19 Revisado 14-10-19 Aprobado 15-01-20 En línea 10-02-20

*Correspondencia

Email: yocana@ucv.edu.pe
Citar como:

Ocaña-Fernández, Y., Valenzuela-Fernández, L., MorilloFlores, J. (2020). La competencia digital en el docente universitario. Propósitos y Representaciones, 8(1), e455. doi: http://dx.doi.org/10.20511/pyr2020.v8n1.455 


\section{Resumen}

Las competencias digitales en el quehacer de la educación superior es un campo muy fecundo que está en constante desarrollo debido a la adecuación de los docentes frente a la demanda de las tecnologías de la información y comunicación y su impacto en el campo educacional. El presente artículo aborda el panorama actual y su dinámica frente a los requerimientos que el docente deberá de desarrollar y las perspectivas de la universidad y los estudiantes. Por otro lado, se aborda la perspectiva de las nuevas tendencias en relación con el panorama los entornos virtuales de aprendizaje y su vinculación en la educación universitaria. Se presenta, además, una discusión final sobre las posibilidades del desarrollo de las competencias en el quehacer académico superior.

Palabras clave: Competencia digital; Tecnologías de la información y comunicación; Entornos virtuales de aprendizaje; Empoderamiento digital.

\section{Summary}

Digital skills in the work of higher education is a very fruitful field that is constantly developing due to the adequacy of teachers in the face of the demand for information and communication technologies and their impact on the educational field. This article deals with the current panorama and its dynamics against the requirements that the teacher must develop and the perspectives of the university and students. On the other hand, the perspective of new trends in relation to the panorama of virtual learning environments and their link in university education, is addressed. In addition, a final discussion is presented on the possibilities of the development of competencies in the higher academic task.

Keyswords: Digital Competence; Information and Communication Technologies; Virtual Learning Environments; Digital Empowerment.

\section{Introducción}

Las tecnologías de la información y la comunicación (TIC) constituyen un pilar básico de la denominada economía digital que en la actualidad es boyante. Su continua evolución ha generado un entorno favorable para nuevos enfoques en relación con los procesos de enseñanza-aprendizaje. La universalización de la internet ha aperturado la aparición de diferentes propuestas: Smart o inteligente, digital, inalámbrica, cibernética o del conocimiento; de estos términos, "Smart" se ha desarrollado y no es ajeno al quehacer académico.

Sobre la llamada cuarta revolución y su impacto apreciado en las sociedades desarrolladas Picatoste, Pérez y Ruesga (2018) manifestaron que dicho impacto también es patente en los estilos de vida de las personas, particularmente debido a sus efectos sobre el mercado y oferta laboral. Sobre estas intempestivas irrupciones producidas por los vertiginosos cambios, también repercuten de manera inmediata acerca de los cambios necesarios en la educación superior para responder a un nuevo panorama mundial altamente cambiante, tal como lo expusieron Turner, Johnston, Kebritchi, Evans y Heflich (2018).

La incorporación en la enseñanza de las tecnologías de la información y las comunicación o TICS en las universidades dentro del plan de las mallas curriculares, como tema transversal de formación, es en sí un elevado desafío, de arduo difícil trabajo; pero muy necesario para preparar a los estudiantes para el éxito en el mercado laboral (Picatoste et al., 2018); ya que los resultados indican que la formación informal en las TICs favorece el empleo y la formación en gestión informática, por lo que Ivars, Celdrán, Mazón y Perles (2017) lo remarcaron al mencionar la necesidad de gestionar y proporcionar canales de auto entrenamiento o entrenamiento personal formal, de preferencia, para ajustarse a las necesidades y disponibilidad laboral futura debido a los cambios continuos en las economías y los mercados globales. 
Según el informe sacado por la OCDE en el año 2015 en relación a un análisis comparativo de alcance internacional acerca de las capacidades digitales de los estudiantes y sus entornos de aprendizaje, demostró que no hay ninguna vinculación directa y con ello causal entre los criterios de la disposición frente al acceso a las TICs; ya que dicho informe muestra como conclusiones que más que el alumno, son las características del docente lo que va a repercutir a manera de factor contextual frente a los recursos (Area, Hernández \& Sosa, 2016).

El empleo de las tecnologías, resultan positivas y su repercusión es trascendente en el campo de la educación en todos sus niveles, ya que su trascendencia está a la par de sus novedades y capacidad de empleo, que según Englund, Olofsson y Price (2016, p. 75) lo referenciaron en base a "The consequences of these differing approaches lie in the manner in which they influence how technology is used to facilitate learning". [Las consecuencias de estos enfoques diferentes radican en la manera en que éstas influyen en cómo se emplea la tecnología para facilitar el aprendizaje]. Sobre este punto cabe recalcar que los profesores nuevos los que son más felxibles a los cambios y que si se adecuan, ello repercutirá de forma positiva en su labor ya que "The ways in which academics conceptualise teaching and learning with technology have significant and interrelated impacts upon their students' experience of learning”. [Las formas en que los académicos conceptualizan la enseñanza y el aprendizaje con tecnología tienen impactos significativos e interrelacionados en la experiencia de aprendizaje de sus estudiantes.] (Kirkwood \& Price, 2013, p. 26). La facilitación de la enseñanza y el aprendizaje mediante el uso de tecnologías como los entornos virtuales se ha expandido rápidamente en la educación superior. (Steils, Tombs, Mawer, Savin-Baden, \& Wimpenny, 2014). Frente a lo anteriormente mencionado, surge la pregunta de cómo los docentes universitarios adoptarán un enfoque basado en la práctica para el uso de las TICs y qué desafíos organizativos y tecnológicos deberán abordarse para apoyar un enfoque basado en la práctica (Baker, Warburton, Hodgkin \& Pascal, 2017)

Según Mykhnenko (2016), la importancia de la aplicación de tecnologías de aprendizaje se considera ahora no solo una parte crucial de la enseñanza-aprendizaje en la educación superior, sino también una evidencia vital de las prácticas innovadoras de enseñanza utilizadas como criterio para la promoción. Los intereses de muchas instituciones de educación superior en el contexto europeo se encuentran en un proceso de trabajo corporativo con visos a la globalización y con ello incrementar su presencia en el mercado online, siempre basado en el empleo de las tecnologías para buscar un promisorio mercado y con ello estar a la vanguardia en lo que respecta a proveer cursos diversos en plataformas virtuales. Dicho aspecto globalizante también es dado a conocer por Mimirinis (2018) quien se refiere al contexto actual de masificación de la población estudiantil a escala mundial, ha conllevado a la necesidad de identificar mejores formas de gestionar el trabajo de los estudiantes y con ello el desempeño de los docentes.

Cabe la pregunta, ¿por qué el requerimiento de competencias digitales para el actual contexto del docente universitario? La respuesta, desde una perspectiva funcional lo brinda Sanaa (2019) al exponer que investigaciones han evidenciado que el cerebro del denominado nativo digital es estructuralmente diferente de los de las generaciones anteriores, asunto que no se encuentra vinculado a la genética, pero es adquirido a partir del entorno digital que los rodea desde su nacimiento; ya que el cerebro al poder procesar las imágenes con una mayor velocidad en relación al texto, va a favorecer, en cierta medida, en las preferencias de sus aprendizajes. Ello, si lo planteamos a la necesidad local, ya de por sí exige que el docente universitario se encuentre frente a un panorama muy diferente bajo el cual fue formado, ya que solo, en su mayor parte, puede replicar el modelo ortodoxo, y deberá virar lo más presto hacia las necesidades y requerimientos cada vez más diversa, cambiantes y crecientes que plantea el escenario de la aplicación de las nuevas tecnologías, para poder empodarse de las mismas y sacar un rédito positivo en su trabajo y desarrollo como profesional. 


\section{Modelos de empleo de los recursos TICs}

Los cambios e innovaciones en todos los sectores del desarrollo humano, incluido en el sector educativo, la mayor parte de las veces son, por lo general, controvertidos, ya que funcionalmente nos conducen a la idea de crisis, inestabilidad e incertidumbre y también progreso y desarrollo, tal como es el caso de su aplicación en el campo educativo donde es promisoria su aplicabilidad tal como lo mencionaron Heinonen, Jääskelä, Häkkinen, Isomäki y Hämäläinen al concebir que "The substantial increase in the use of information and communication technologies (ICT) has raised expectations for their educational potential in higher education" [El aumento sustancial en el uso de las tecnologías de la información y la comunicación (TIC) ha elevado las expectativas de su potencial educativo en la educación superior.] (2019, p. 1). Similar percepción es manifestada por la investigación llevada cabo por Ocaña, Valenzuela y Garro quienes expusieron la radical trascendencia de las tecnologías en el campo de la educación superior al exponer que "En el actual contexto nos encontramos inmersos en una sociedad que se orienta, cada vez más, hacia el proceso de la tecnificación masiva." (p. 537, 2018). La necesidad de empleo de las mismas o de obligada recurrencia puede sostenerse en lo manifestado por Carrillo, Cascales y Valero al mencionar que la "...revolución tecnológica ha llegado a las aulas, bien de la mano de los alumnos, de los propios docentes o como reclamo de la sociedad, y por tanto no podemos obviarla" (p.1,2018). Sobre este entorno, descrito ampliamente en la literatura, Area et al. (2016) nos mencionaron que dicho predicamento se desliza sobre tendencias innovadoras dentro del campo del empleo de las novedosas tecnologías de la información, lo que se va a traducir como patrón o perfil de empleo de los recursos TICs, que en palabras de Area et al. (2018) se tendrían como factores limitantes al escaso apoyo a los procesos de enseñanza en relación a un uso efectivo de esos recursos, lo cual estaría, hasta cierta medida, mediado por la edad de los docentes, el género, la competencia digital, la actitud hacia las TICs y la disposición hacia el cambio y la innovación. La apremiante necesidad de cambios sustantivos en la educación universitaria del contexto regional es el punto álgido a fin de generar "una completa transformación de los estándares estereotipados de los modelos educacionales universitarios y posicionarlos con un rango de empoderamiento digital que es lo que esta nueva generación requiere" (Ocaña et al., 2018, p. 550).

Un aspecto de gran relevancia para el docente universitario en relación con las nuevas tecnologías es el adaptarse y mimetizarse con las mismas, lo que en sí no es una tarea de fácil ejecución ya que entre otras cosas demandará de los mismos su adecuación a la alfabetización digital y todo cuanto ello demande. (Carrillo et al., 2018; Ocaña et al., 2018). Por otro lado, Ottenbreit, Liao, Sadik y Ertmer (2018) sostuvieron que algunos investigadores han sugerido que, si los maestros manejan conocimientos lo suficientemente sólidos, podrán superar las barreras y con ello integrar con éxito la tecnología a su práctica. Voce y Jenkins (2016) mencionaron que, si los programas de formación docente se orientan a formar docentes preparados para emplear la tecnología, podrán superar las barreras externas o de primer orden existentes. Por su parte Espinosa, Porlán y Sánchez (2018) ponen en evidencia el considerar a "las tecnologías como recursos de gran relevancia en el contexto de la Universidad" (p. 6). Si bien es cierto que en sus inicios muchos docentes a nivel superior se mostraban reacios a la aplicación de las nuevas tecnologías debido a la retahíla de ser reemplazados por las mismas en la educación, tal como fue expuesto por Greener (2018), esbozando una especie de panorama apocalíptico al respecto; en la actualidad, en palabras del mismo autor es otro ya que "Today we are much further on: the field of technology-enhanced learning is fertile and verywell populated." [Hoy vamos mucho más allá: el campo del aprendizaje mejorado por la tecnología es fértil y está muy poblado.] (p. 856)

Un aspecto que se desarrolla de forma vertiginosa es la generación de las App como herramientas que facilitan el aprendizaje dentro del marco del denominado Mobile Learning, según lo expuesto por Carrillo et al. (2018) así como Espinosa et al., (2018) al poner de manifiesto su aplicación en la enseñanza de lenguas extranjeras y su repercusión en el contexto español, con muy buenos resultados. Sin duda los aplicativos móviles trascienden la barrera del tiempo y el espacio, facilitando la diversificación de contenidos, los cuales podrían, en muchos casos, orientarse a diversas materias que lo requieran, claro que para ello habrá que denotar la plataforma en la cual 
deberá generarse la aplicación, su versatilidad, y entre todo ello la asequibilidad den su diseño que genere un entorno amigable con el usuario. (Mena, 2018). Un punto a favor de la aplicación de estas tecnologías es que puede ser constantemente mejorada y actualiza, y si es que esta tendencia sigue de forma creciente podrá "favorecer el desarrollo de estrategias pedagógicas de cara a la planificación e implementación de la enseñanza." (Espinosa et al., 2018, p. 13). Sin embargo, el proceso de adopción e implementación de estas tecnologías móviles sigue siendo una tarea compleja no solo en el debate académico sino también entre los profesionales (Ben \& Boonstra, 2019).

Investigadores como proponen repensar los modelos de enseñanza, bajo la óptica de las novedosas tecnologías que en su dinamismo son cada vez más comunes respecto de su empleo en la educación superior ya que ello puede "involve ICTs in order to obtain more relevant and useful information and to introduce modern teaching methods to study a certain topic or solve a specific problem." [involucrar a las TICs para obtener información más relevante y útil e introducir métodos de enseñanza modernos para estudiar un determinado tema o resolver un problema específico.] (Gorghiu, Gorghiu \& Pascale, 2018, p. 3). Al respecto se subraya la posibilidad del desarrollo de la educación en línea, como componente mediador de la $e$-educación ya esto ofrece la posibilidad de automatizar los diversos procesos que, en el ejercicio docente tradicional, tenían que ser asumidos y realizados por el profesor. Y en este panorama relacionado a las TICs, las herramientas de la Web 2.0 y sus recursos en línea, así como el creciente empleo de la multimedia, son elementos ya de uso bastante extendido en las aulas universitarias; pero que también pone en evidencia prometedores visos de novedosas formas de interacción que superan los límites del claustro ya que disponibilidad trascienden el tiempo y espacio (Ocaña, et al., 2018), y que además "The inclusion of the ICT resources in the teaching strategies constitutes a variable that favours the growth of the learning efficiency, having a positive impact on the student, but also on the teacher's activities." [La inclusion de los recursos de las TIC en las estrategias de enseñanza constituye una variable que favorece el crecimiento de la eficiencia del aprendizaje, teniendo un impacto positivo en el alumno, pero también en las actividades del profesor.] (Gorghiu, et al., 2018, p. 6)

Los recursos de las TICs pueden adaptarse a los requerimientos de aprendizaje de los estudiantes, pero también a las necesidades de enseñanza de los maestros. El empleo de las TICs en los procesos de enseñanza-aprendizaje genera en los estudiantes una mayor atención a lo que se enseña, incrementando su receptividad e interactividad al mismo tiempo que se estimula su creatividad. (Martínez, Mauricio y Lugo, 2016). Por otro lado, el empleo de las TICs coadyuva al desarrollo de habilidades digitales, para trabajar en colaboración en proyectos y para resolver problemas más rápido y con gran precisión. Por todo ello las "ICTs can be adapted to the students' learning needs, but also to the teachers' teaching needs" [Las TIC se pueden adaptar a las necesidades de aprendizaje de los estudiantes, pero también a las necesidades de enseñanza de los profesores] (Gorghiu et al., 2018, p. 9).

\section{Competencias digitales docentes}

La tendencia a nivel de la docencia universitaria es creciente en relación con su adecuación al plano digital, tal como lo remarcan Espinosa et al., (2018) al exponer su punto de vista sobre la competencia digital, ya que para dichos autores ello significa ir más allá de entender el uso de las tecnologías, subrayando la trascendencia del dantesco impacto de las mismas dentro del universo digital. A nivel aplicativo, lo anterior puede esgrimirse en los términos planteados por Davies (2017, p.3) quien expuso "Everyone needs to acquire a minimum set of competences in order to learn, work and achieve fulfilment in a knowledge-driven society and economy" [Todos deben adquirir un conjunto mínimo de competencias para aprender, trabajar y alcanzar el cumplimiento en una sociedad y economía impulsadas por el conocimiento], dando relevancia a la repercusión de las competencias en el contexto actual.

Según lo manifestado por Carrillo et al. (2018, p. 3) debe entenderse por digital lo referente "a la convergencia de las tecnologías de computación y de comunicación para generar medios que 
estén disponibles en Internet, la Web o en cualquier plataforma que los incluya”, y que, en palabras de Espinosa et al. (2018) la competencia digital, desde una perspectiva funcional para el docente universitario, significa su aproximación a una adecuada integración de las TICs en su función docente.

Espinosa et al. (2018) mencionaron su apreciación sobre la competencia profesional docente se vincula directamente con la competencia digital, que de forma funcional la adquisición de la misma está directamente relacionado con su nivel de profesionalización. Además, dichos autores expusieron la importancia de los docentes como agente clave de la vinculación profesional orientado al cambio cualitativo en el nivel superior, cuyo accionar está anexado indisolublemente al empleo de las TICs, para lo cual demostrar el nivel requerido respecto de las competencias digitales, es lo que deberá caracterizar su ejercicio profesional docente. Según este enfoque la experticia del docente de nivel superior se vincula al hecho no solo de su capacidad como docente e investigador, sino que exige del mismo una adecuación constante frente a las cada vez más diversas tecnologías. Sobre ello cabe la apreciación certera que hacen Englund, Olofsson y Price (2016) al poner de manifiesto la necesidad de investigar los cambios y curso del desarrollo sobre las concepciones y enfoques de parte de los docentes sobre el uso de las tecnologías, ya que "....as the digital tools we use and the online environments in which we live and learn proliferate." [a medida que proliferan las herramientas digitales que utilizamos y los entornos en línea en los que vivimos y aprendemos] (Greener, 2018, p. 856).

Veamos, por otro lado, modelos desarrollados sobre el campo de las competencias a nivel de la docencia superior tal como lo mencionan Englund et al. (2016) al realizar un certero análisis de la ambiciosa estrategia de Lisboa al 2010, propuesta por la Unión Europea, la cual se proponía sustentarse en una economía basada en el conocimiento con visos a orientar su desarrollo económico sostenible, de las cuales se rescata como competencia clave, la competencia digital dentro del marco por ellos considerado dentro del rubro de las nuevas tecnologías de información y comunicación. A pesar de sus esfuerzos, el proyecto alcanzó ciertos atisbos, ya que tuvo que ser reconsiderado en función de los enormes cambios en la economía global de mercado que exigía mayores ajustes y tras ello la reestructuración de lo proyectado. Pues bien, el proceso no se detuvo y es prueba de ello la investigación desarrollada por Gorghiu et al. (2018) que reveló lo limitado de las capacidades del ciudadano europeo para poder sacar el máximo provecho de empleo de las tecnologías digitales. En palabras de Espinosa et al. (2018) se tiene expresamente que debe generarse una redefinición de las competencias del docente universitario para incorporar la competencia digital bajo una perspectiva holística que logre abarcar todos los aspectos y relevancias de esta.

Al realizar un importante estudio a nivel de sus ciudadanos, la Unión Europea evidenció que muchos de ellos tienen capacidades limitadas para explotar todo el potencial de las tecnologías digitales en su vida cotidiana. Debido a este resultado, la Comisión Europea propuso el Marco Europeo de Competencia Digital para Ciudadanos, conocido como DigComp, (basado en el modelo conceptual DigComp 2.0), que ilustra ocho niveles de competencia y ejemplos de uso, aplicados al campo de aprendizaje y empleo, que identifica 21 competencias en cinco áreas clave, que se detalla en la figura 1 .

Resolver problemas técnicos. Identificar necesidades y respuestas tecnológicas. Uso creativo de tecnologías digitales. Identificar brechas de competencia digital.
Navegación, búsqueda y filtrado de datos, información y contenido digital. Evaluación de datos, información y contenido digital.

Gestión de datos, información y contenido digital.

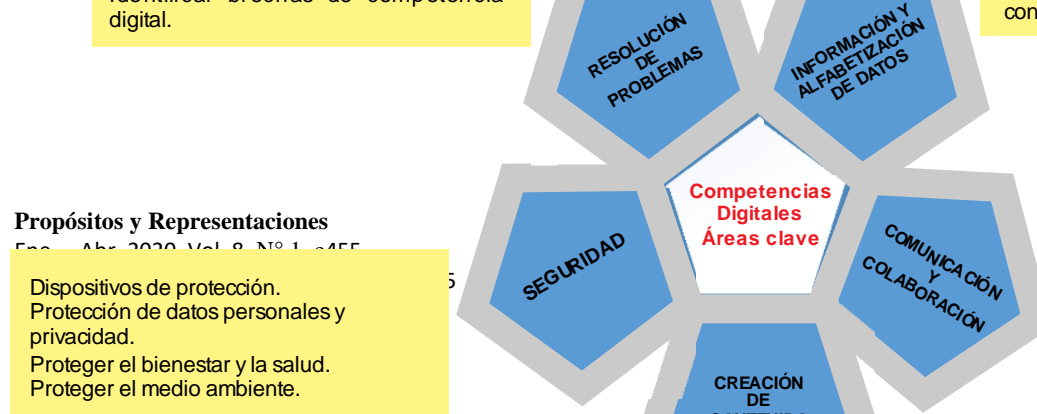

Interactúar a través de tecnologías digitales. Compartir a través de tecnologias digitales. Involucrarse en la ciudadanía a través de tecnologías digitales.

Colaborando a través de tecnologí 
Figura 1. Dig Comp's cinco áreas clave y 21 competencias. (Tomado y adaptado de Gorghiu, Gorghiu \& Pascale, 2018).

Los cambios en relación a las competencias digitales, es producto del cada vez avasallador desarrollo de nuevas y mejores tecnologías, tal como lo planteó Sanaa (2019) al manifestar que inclusive un denominado nativo digital está supeditado a convertirse en un inmigrante digital, cada vez que una nueva tecnología aparece y supera a la anterior, ello evidencia una problemática direccionada a los docentes, que no solo deberá encontrarse actualizado sino que pueda desarrollar un trabajo solvente para poder atender adecuadamente las perspectivas de los estudiantes actuales, muy afines al uso de la tecnología. Ciertamente, existen diferencias intrageneracionales cuando se trata de aprender nuevas tecnologías, que se podrían explicar como: "It is natural that new generations learn faster than their predecessors, but this could be explained by how often new generations use technology and not because they are innately technology-savvy.'[Es natural que las nuevas generaciones aprendan más rápido que sus predecesores, pero esto podría explicarse por la frecuencia con la que las nuevas generaciones usan la tecnología y no porque sean conocedores de la tecnología de manera innata.] (Sanaa, 2019, p. 4).

\section{Los entornos virtuales de aprendizaje}

No muchas veces los docentes se encuentran en paridad competitiva para poder hacer frente a la realidad del empleo de las nuevas tecnologías, lo que se traduce en desmedro como profesional en función de la realidad que demanda las adecuaciones, ya que "However, when teachers are inexperienced with teaching with technology, they frequently base their initial development of teaching on their overall pedagogical beliefs" [Sin embargo, cuando los maestros no tienen experiencia en la enseñanza con tecnología, con frecuencia basan su desarrollo inicial de la enseñanza en sus creencias pedagógicas generales.] (Englund et al., 2016, p. 75). Desde otra óptica se tiene que la percepción de los estudiantes, a nivel superior, es muy incisiva sobre este aspecto, ya que al respecto el trabajo longitudinal de Englund et al. (2016) llevado a cabo por 10 años para los niveles de pre y posgrado respecto a la entrega de materiales y administración de cursos digitales, un entorno virtual de aprendizaje, hallando resultados muy satisfactorios al respecto en relación a la aceptación hacia los estudiantes y la adecuación de los docentes a las nuevas tecnologías que facilitó su labor.

Asumir los retos y cambios por parte de los docentes universitarios es tendencia, ya que el llamado es para todos ellos, más no todos ellos serán los escogidos, ya que el dilema funcional se encuentra entre aquellos docentes que manejan una visión ortodoxa de la enseñanza universitaria, tanto en pregrado como posgrado, que en cierta manera se encuentra en notable desventaja en relación a la nueva generación de docentes que apuestan por el empleo cada vez más constante de las nuevas tecnologías y que es en este punto donde, los últimos, salen airosos de tal contienda, lo que se puede interpretar como "Novice teachers are more malleable in terms of their ability to change 
their conceptions and approaches". [Los maestros novatos son más maleables en términos de su capacidad para cambiar sus concepciones y enfoques.] (Englund et al., 2016, p. 84). Aunque los maestros novatos en un inicio desarrollan su experticia docente bajo el enfoque tradicional, muchos de ellos han virado de aquella situación de emulación de los colegas con más años de experiencia, ya que son más maleables en términos de su capacidad para cambiar sus concepciones y enfoques. Gorghiu, Gorghiu y Pascale (2018) mencionaron que los docentes empezaron a emplear las computadoras y diversas herramientas de las TICs para el desarrollo de lecciones de informática, en un inicio, y posteriormente, dichas tecnologías fueron involucradas en el proceso de capacitación de otras disciplinas. Además, cabe acotar que la internet provee de diversos recursos de información en las aulas, generando plataformas de comunicación abierta y rápida entre maestros y alumnos. Una lección que explota las ventajas de la internet y las tecnologías multimedia puede considerarse un formato de maestro virtual, mostrando un mayor apoyo a través del desarrollo de lecciones previas bajo un soporte multimedia, actividades prácticas asistidas por computadora, instrumentos de evaluación e instrumentos de aprendizaje cooperativo en línea.

Desde otra perspectiva Greener (2018) nos manifestó que el tema de las tecnologías es muy discutido en los entornos académicos en relación al proceso enseñanza-aprendizaje; pero son recursos que muy poco se emplean, o que en muchos casos de ser utilizados, nos e logra aprovechar la potencialidad completa de los mismos debido al desconocimiento o falta de capacitaciones adecuadas que le faciliten al docente universitario lograr un nivel aceptable en relación al empleo con un muy aceptable nivel de performance. Si tomamos en cuenta el trabajo desarrollado por Heinonen en relación a la educación superior en Finlandia, muestran también en parte las deficiencias en docencia superior al exponer que "the present findings suggest that university teachers do not utilize research evidence when developing new teaching methods involving technology." [Los hallazgos actuales sugieren que los docentes universitarios no utilizan evidencia de investigación cuando desarrollan nuevos métodos de enseñanza que involucran tecnología.] (2019, p. 12).

Es cierto que existe una brecha entre el saber y el hacer, pero los diversos trabajos de investigación y la literatura académica demuestran los múltiples beneficios pedagógicos de un adecuado diseño de aprendizajes que incorporan la tecnología generando eficiencia en el aprendizaje. Si se asume el empleo de las tecnologías como los entornos de aprendizaje virtual (VLE) a nivel de la docencia superior, ello está ofreciendo una serie de ventajas, tales como llegar a la mayor cantidad de usuarios, el introducir flexibilidad en la programación y el ritmo de aprendizaje, y mejorar los procesos de supervisión y la estandarización de la enseñanza. Sobre este panorama Cahillane, MacLean y Smy (2018) desarrollaron una investigación en relación al empleo de los entornos virtuales de aprendizaje, hallando en dicho estudio que la aplicación de cursos virtuales son significativamente muy acertados, ya que se pueden explotar una serie de recursos que respecto de una clase típica no se podría lograr; y lo resumen en lo siguiente: "This suggests that the requirement to understand the cognitive aspects of the technical skills required to produce online learning content will be amplified alongside pedagogical considerations." [Esto sugiere que el requisito para comprender los aspectos cognitivos de las habilidades técnicas requeridas para producir contenido de aprendizaje en línea se amplificará a la par de las consideraciones pedagógicas.] (Cahillane et al., 2018, p. 10).

\section{Las aplicaciones de los espacios de aprendizaje con tecnología mejorada}

Los estudios acerca del rubro de los espacios de aprendizaje potenciados por la tecnología se han convertido, en los últimos años, en el foco de atención en el campo de la educación según lo manifestado por Xu, Chiu y Ye (2019). Sin embargo, no existe una definición clara de espacio de aprendizaje o espacio respaldado por tecnologías. De forma general, un espacio de aprendizaje es considerado como un entorno de aprendizaje generado y orientado a inspirar el interés de los aprendizajes por parte de los estudiantes, alentar el compromiso de los mimos y con ello fortalecer una interacción muy dinámica entre maestros y estudiantes. (Xu et al. 2019; Imms y Byers, 2016; Tondeur, Bruyne, Driessche, Mckenney y Zandvliet, 2015) Los espacios de aprendizaje, por su 
naturaleza, poseen muchas funciones como diseño flexible, escritorios móviles, espacio multipantalla, interconectividad e intercambio de información y un sistema ubicuo de apoyo continuo del aprendizaje, se caracterizan por la vinculación del espacio físico y el espacio virtual. Los espacios de aprendizaje con tecnología mejorada se orientan a enfatizar la enorme importancia a la tecnología de materiales, particularmente el efecto de la tecnología de la información moderna, en el proceso de construcción de espacios de aprendizaje, sobre la base de considerar los factores de planificación del espacio, entorno físico, mobiliario, entre otros (Tondeur, et al., 2015).

En la actualidad, diversos estudios están relacionados con el alto impacto de los espacios de aprendizaje mejorados por la tecnología en el rendimiento académico, así como el comportamiento de enseñanza de los estudiantes; indicando la mayoría de dichos estudios resultados positivos, es decir, que los espacios de aprendizaje mejorados por la tecnología pueden facilitar el compromiso y la interacción de manera efectiva de los estudiantes (Xu et al., 2019).

Si bien la prevalencia de las ciencias del aprendizaje y el constructivismo han impuesto nuevas demandas en los espacios de aprendizaje, las nuevas tecnologías, representadas por las tecnologías de la información y la comunicación (TICs), están permitiendo generar nuevos entornos para reestructurar los denominados espacios de aprendizaje; ya que se han generado sendos estudios sobre los espacios de aprendizaje potenciados por la tecnología que, actualmente, es tendencia en el campo de la educación superior. (Gorghiu et al., 2018; Ottenbreit-Leftwich, Liao, Sadik \& Ertmer, 2018; Herro, 2015).

Actualmente, muchos estudios en el campo están relacionados con el impacto de los espacios de aprendizaje mejorados por la tecnología en el rendimiento académico y el comportamiento docente de los estudiantes, y la mayoría de los estudios indicar resultados positivos (Xu et al., 2019). De manera similar es lo referido por Imms y Byers (2016) en su investigación de la aplicación de espacios de aprendizaje mejorados por tecnología en el caso de la enseñanza de las ciencias físicas, generando una aceptación muy alta por parte de docentes y estudiantes que facilitó los procesos de aprendizaje, ya que se aplicaron plataformas en tercera dimensión lo que permitió un análisis más fecundo de los procesos físicos, y aunque es uno de los primeros intentos, los resultados, tal como lo mencionan, no son casualidad y que se espera que se generen nuevas investigaciones para afianzar el campo aplicativo de esta prometedora tecnología.

\section{Conclusiones}

De acuerdo con Ottenbreit et al. (2018) la investigación futura debería centrarse en desarrollar asociaciones estrechamente vinculantes de investigación-práctica para identificar los mejores mecanismos para apoyar el crecimiento de los docentes frente a las nuevas tecnologías. De similar tendencia es lo expuesto por Ocaña et al. (2018) lo señalan al referirse que la vanguardia que se genere en el campo del desarrollo de las capacidades digitales docentes sustentadas en las nuevas tecnologías brindará un salto cualitativo acorde a la exigencia imperante por el sistema global de producción. Este aspecto resulta ser de gran repercusión, dado el hecho por el que muchos países buscan los mecanismos más eficaces para lograr acrecentar el empleo de las tecnologías, en especial desde el plano educacional, siendo la formación universitaria la prioridad para los países con mayor desarrollo tecno-económico ya que "... a fundamental transformation of education and training is required, in order to deliver the needed knowledge and skills for the societal development, employment and involvement in the society issues" [... se requiere una transformación fundamental de la educación y la formación, a fin de proporcionar los conocimientos y habilidades necesarias para el desarrollo social, el empleo y la participación en los problemas de la sociedad.] (Gorghiu et al., 2018, p. 184). También cabe notar lo manifestado por Ventura, Cuberes y Corral (2018) quienes dan a conocer que "diversas universidades europeas y norteamericanas ya incluyen programas de estudio sobre medios digitales e interactivos en su oferta educativa" (p. 334), dejando en evidencia que la tendencia en el campo de la digitalización del entorno académico universitario es una carrera en pleno apogeo. De forma similar es la perspectiva de Joop (2019) en relación al desarrollo de las 
competencias digitales y su aplicación a la actividad docente que en su punto de vista, genera cambios más que significativos en la experticia docente que van a repercutir en su accionar, inclusive en la mediación de entornos de evaluación de resultados, ya que las perspectivas del e-learning proponen un panorama creciente promisorio que el investigador explicó como "To advance the dissemination of best practice it is essential that experiences with authentic, technology-enhanced assessment, such as this, are shared more widely." [Para avanzar en la difusión de las mejores prácticas, es esencial que las experiencias con una evaluación auténtica y mejorada por la tecnología, como esta, se compartan más ampliamente.] (Joop, 2019, p. 14). De forma similar la investigación de Sanaa (2019) en Emiratos Árabes concuerda con dicha perspectiva acerca de lo significativo de las competencias digitales en labor docente en la educación superior al manifestar "use of technology inside the classroom is far less than the students' expectations." [ El uso de la tecnología dentro del aula es mucho menor que las expectativas de los estudiantes.] (p. 11)

"En definitiva, en las universidades del siglo XXI, el docente ha de dejar de ser el controlador del acceso a la información del alumno y pasar a ser el alumno el último responsable de ello" (Espinosa et al., 2018, p. 9). Es recomendable que los docentes universitarios sean conscientes del impacto positivo que generan las TICs en el aprendizaje y la enseñanza, ofreciéndoles nuevos posibles horizontes, con resultados positivos en su desempeño y el logro deseado. Ello no es una tarea nada sencilla ya que "Innovation with digital media to promote learning in schools is fraught with challenges." [La innovación con medios digitales para promover el aprendizaje en las escuelas está llena de desafíos] (Herro, 2015, p. 2); pero sus perspectivas pueden ser muy alentadoras tal como lo manifiestan Heinonen et al. (2019, p. 1) al sostener "In the current and future digital world, technology-enhanced teaching and learning must be developed in HE to guarantee that university students will obtain high-level skills for the future working life". [En el mundo digital actual y futuro, la enseñanza y el aprendizaje mejorados por la tecnología deben desarrollarse en la educación superior para garantizar que los estudiantes universitarios obtengan habilidades de alto nivel para la vida laboral futura].

Los denominados nativos digitales, de por sí ya es costumbre para ellos convivir con la tecnología; pero a su vez, los cambios en el campo tecnológico son tan raudos que, si dicho grupo no se preparan para hacerlo frente, pronto sucumbirán ante tal ineluctable avance, volviéndose por ende obsoletos, (Picatoste et al., 2018) y ello no es producto de un vaticinio negativo, sino que es preclaro concebir tal escenario en función de los trascendentales cambios de las nuevas tecnologías y su repercusión a diversos niveles clave. Por lo manifestado, existe la necesidad imperativa de una formación continua y la necesidad de instituciones y empresas de promover cursos de formación para sus empleados si es que queremos como nación no hacer frente ante tan dantesca ola de cambios, sino navegar sobre la misma y correr a la par. Por todo ello, las instituciones de educación superior, en especial las universitarias, tienen un papel decisivo en la generación, promoción y gestión del conocimiento y la innovación tecnológica, pero este nuevo escenario, será posible cunado el cuerpo docente se encuentre a la altura del desafío que enmarca el reto tecnológico que los nuevos tiempos reclaman.

\section{Referencias}

Area, M., Hernández, V., \& Sosa, J. (2016). Modelos de integración didáctica de las TIC en el aula. Comunicar, 24(47), 79-87. doi: https://doi.org/10.3916/C47-2016-08

Baker, S., Warburton, J., Hodgkin, S., \& Pascal, J. (2017). The New Informational Paradigm: Developing Practice-Led Approaches to the Use of Mobile ICT in Social Work. The British Journal of Social Work, 1-19. doi: https://doi.org/10.1093/bjsw/bcx124

Ben, S., \& Boonstra, J. (2019). The development and implementation of a mobile application in human services. Journal of Technology in Human Services, 37(2-3), 159-183. doi: https://doi.org/10.1080/15228835.2019.1626788 
Carrillo, M., Cascales, A., \&Valero, A. (2018). Apps para el aprendizaje de idiomas en la Universidad de Murcia. Revista de Educación a Distancia, 58(13), 1-18. Recuperado de http://www.um.es/ead/red/58/carrillo_et_al.pdf

Cahillane, M., MacLean, P., \& Smy, V. (2018). Novel application of a predictive skill retention model to technical VLE content production skills among Higher Education teachers: a case study. Interactive Learning Environments, (1) 1-13. doi: https://doi.org/10.1080/10494820.2018.1474231

Davies, H. (2017). Competence-Based Curricula in the Context of Bologna and EU Higher Education Policy. Pharmacy, 5(17). doi: https://doi.org/10.3390/pharmacy5020017

Englund, C., Olofsson, A. D., \& Price, L. (2016). Teaching with technology in higher education: understanding conceptual change and development in practice. Higher Education Research \& Development, 36(1), 73-87. doi: https://doi.org/10.1080/07294360.2016.1171300

Espinosa, M., Porlán, I., \& Sánchez, F. (2018). Competencia digital: una necesidad del profesorado universitario en el siglo XXI. Revista de Educación a Distancia, 56 (7), 1-22. Recuperado de http://www.um.es/ead/red/56/prendes_et_al.pdf

Greener, S. (2018). The knowing-doing gap in learning with technology. Interactive Learning Environments, 26(7), 856-857. doi: https://doi.org/10.1080/10494820.2018.1510155

Gorghiu, G.; Gorghiu, L., \& Pascale, L. (2018). Enriching the ICT competences of university students - A key factor for their success as future teachers. Journal of Science and Arts, 1(42), 183-190.

Haris, A., Washizaki, H., \& Fukazawa, Y. (2017). Utilization of ICTs in quality assurance and accreditation of higher education: Systematic literature review. IEEE 6th International Conference on Teaching, Assessment, and Learning for Engineering (TALE). doi: https://doi.org/10.1109/tale.2017.8252361

Heinonen, K., Jääskelä, P., Häkkinen, P., Isomäki, H., \& Hämäläinen, R. (2019). University Teachers as Developers of Technology-Enhanced Teaching-Do Beliefs Matter? Journal of Research on Technology in Education, 1-17. doi: https://doi.org/10.1080/15391523.2018.1564894

Herrero, P., Conde, J., Tapia, A., \& Varona, D. (2019). La credibilidad de las noticias en Internet: una evaluación de la información por estudiantes universitarios, Cultura y Educación, 31(2), 407-435. doi: https://doi.org/10.1080/11356405.2019.1601937

Herro, D. (2015). Sustainable Innovations: Bringing Digital Media and Emerging Technologies to the Classroom. Theory Into Practice, 54(2), 117-127. doi: https://doi.org/10.1080/00405841.2015.1010834

Hoel, T., \& Chen, W. (2018). Privacy and data protection in learning analytics should be motivated by an educational maxim-towards a proposal. Research and Practice in Technology Enhanced Learning, 13(20), 1-14. doi: https://doi.org/10.1186/s41039-018-0086-8

Imms, W., \& Byers, T. (2016). Impact of classroom design on teacher pedagogy and student engagement and performance in mathematics. Learning Environments Research, 20(1), 139152. doi: https://doi.org/10.1007/s10984-016-9210-0

Ivars, J., Celdrán, M., Mazón, J., \& Perles, Á. (2017). Smart destinations and the evolution of ICTs: a new scenario for destination management? Current Issues in Tourism, 1-20. doi: https://doi.org/10.1080/13683500.2017.1388771

Jopp, R. (2019). A case study of a technology enhanced learning initiative that supports authentic assessment. Teaching in Higher Education, 1-17. doi: https://doi.org/10.1080/13562517.2019.1613637

Kirkwood, A., \& Price, L. (2013). Technology-enhanced learning and teaching in higher education: what is "enhanced" and how do we know? A critical literature review. Learning, Media and Technology, 39(1), 6-36. doi: https://doi.org/10.1080/17439884.2013.770404

Mancillas, L. \& Brusoe, P. (2016). Integrating media technology in the political science classroom. Journal of Political Science Education, 12(4), 375-386. doi: https://doi.org/10.1080/15512169.2015.1096792

Martínez, G., Mauricio, A., \& Lugo, C. (2016). Formación Docente en Tic Con El Centro De Innovación Educativa Cier-Sur. Revista Trilogía, 8(14), 65-80. 
McKnight, K., O’Malley, K., Ruzic, R., Horsley, M., Franey, J. J., \& Bassett, K. (2016). Teaching in a Digital Age: How Educators Use Technology to Improve Student Learning. Journal of Research on Technology in Education, 48(3), 194-211. doi: https://doi.org/10.1080/15391523.2016.1175856

Mena, N. (2018). Redes sociales, Internet de las cosas y competencias digitales de profesores e investigadores en Medicina. Revista Cubana de Educación Médica Superior, 32(2), 1-16.

Mimirinis, M. (2018). Qualitative differences in academics' conceptions of e-assessment. Assessment \& Evaluation in Higher Education, (1) 1-16. doi: https://doi.org/10.1080/02602938.2018.1493087

Mykhnenko, V. (2016). Cui bono? On the relative merits of technology-enhanced learning and teaching in higher education. Journal of Geography in Higher Education, 40(4), 585-607. doi: https://doi.org/10.1080/03098265.2016.1217832

Ocaña, Y., Valenzuela, L., \& Garro, L. (2019). Inteligencia artificial y sus implicaciones en la educación superior. Propósitos y Representaciones, 7(2), 536-568. doi: https://doi.org/10.20511/pyr2019.v7n2.274

Orin, C. (2019). Curriculum Transformation: From Didactic to Competency-Based Programs in Pharmaceutical Medicine. Frontiers in Pharmacology, 10(1), 278. doi: https://doi.org/10.3389/fphar.2019.00278

Ottenbreit, A., Liao, J., Sadik, O., \& Ertmer, P. (2018). Evolution of Teachers' Technology Integration Knowledge, Beliefs, and Practices: How Can We Support Beginning Teachers Use of Technology? Journal of Research on Technology in Education, 1-23. doi: https://doi.org/10.1080/15391523.2018.1487350

Patton, R., \& Buffington, N. (2016). Keeping up with our students: The evolution of technology and standards in art education. Arts Education Policy Review, 117(3), 1-9. doi: https://doi.org/10.1080/10632913.2014.944961

Peters, M., Jandrić, P., \& Hayes, S. (2018). The curious promise of educationalising technological unemployment: What can places of learning really do about the future of work? Educational Philosophy and Theory, 1-13. doi: https://doi.org/10.1080/00131857.2018.1439376

Picatoste, J., Pérez, L., \& Ruesga, S. (2018). A new educational pattern in response to new technologies and sustainable development. Enlightening ICT skills for youth employability in the European Union. Telematics and Informatics, 35(4), 1031-1038. doi: https://doi.org/10.1016/j.tele.2017.09.014

Sanaa, A. (2019): How technology has shaped university students' perceptions and expectations around higher education: an exploratory study of the United Arab Emirates, Studies in Higher Education, 1-14. doi: https://doi.org/10.1080/03075079.2019.1617683

Steils, N., Tombs, G., Mawer, M., Savin-Baden, M., \& Wimpenny, K. (2014). Implementing the liquid curriculum: the impact of virtual world learning on higher education. Technology, Pedagogy and Education, 24(2), 155-170. doi: https://doi.org/10.1080/1475939X.2014.959454

Tondeur, J., Bruyne, E., Driessche, M., Mckenney, S., \& Zandvliet, D. (2015). The physical placement of classroom technology and its influences on educational practices. Cambridge Journal of Education, 45(4), 537-556. doi: https://doi.org/10.1080/0305764x.2014.998624

Turner, P., Johnston, E., Kebritchi, M., Evans, S., \& Heflich, D. (2018). Influence of online computer games on the academic achievement of nontraditional undergraduate students. Cogent Education, 5(1), 1-16. doi: https://doi.org/10.1080/2331186X.2018.1437671

Ventura, R., Cuberes, C., \& Corral, A. (2018). Comunicación Digital Interactiva: valoración de profesionales, docentes y estudiantes del área de la comunicación sobre las competencias académicas y los perfiles profesionales. Revista Latina de Comunicación Social, (73), 331351. doi: https://doi.org/10.4185/RLCS-2018-1258

Xu, Y., Chiu, C.-K., \& Ye, X. (2019). Understanding the use of technology-enhanced learning spaces in Hong Kong: an exploratory study. Asia Pacific Journal of Education, 1-20. doi: https://doi.org/10.1080/02188791.2019.1598846 
Zhang, J., Zhang, X., Jiang, S., Ordóñez, P., \& Sun, Y. (2018). Mapping the study of learning analytics in higher education. Behaviour \& Information Technology, 37(10-11), 1142-1155. doi: https://doi.org/10.1080/0144929X.2018.1529198 